\title{
ONE DIMENSION HIGHER OF THE WORD PROBLEM
}

\author{
A. SINAN ÇEVIK
}

\begin{abstract}
Just as a group presentation $\mathscr{P}$ can be regarded as a 2-complex with a single 0 -cell, so we can consider a 3-complex with a single 0-cell, known as a 3-presentaton. In this paper, by using a geometric way, called spherical pictures, we show that there exist a finite 3-presentation which has unsolvable generalised identity problem which can be thought as one-dimension higher of the word problem.
\end{abstract}

\section{Introduction}

Let $G$ be a group defined by a presentation

$$
\mathscr{P}=\langle\mathbf{x} ; \mathbf{r}\rangle .
$$

Therefore we have a free group $F(\mathbf{x})$ on $\mathbf{x}$, a normal closure set $N$ of $\mathbf{r}$ in $F(\mathbf{x})$ and a quotient group, that is the group defined by $\mathscr{P}, G(\mathscr{P})=F(\mathbf{x}) / N$ such that $G=G(\mathscr{P})$ for finite presentation $\mathscr{P}$. We recall that a typical element of $G(\mathscr{P})$ is represented by $\bar{W}=[W] N$ where $W$ is a word on $\mathbf{x}$ and $[W]$ is the free equivalence class of $W$.

The word problem is the question of asking for the existence of an algorithm to determine of an arbitrary word $W$ on $\mathbf{x}$ whether or not $\bar{W}=1$ in $G$.

Let $H$ be a finitely generated subgroup of $G$. Then the subgroup word problem for $H$ in $G$ is the problem of deciding for an arbitrary word $W$ on $\mathbf{x}$, whether or not $W$ defines an element of $H$. It is clear that if $H$ is trivial then the subgroup word problem is just simply the word problem.

If we think $\mathscr{P}$ as a finite connected 2-complex then the word problem for $\mathscr{P}$ is the problem of determining the existence of algorithm to decide whether for any arbitrary element of the first homotopy group $\pi_{1}(\langle\mathbf{x}\rangle)$, its image under the inclusion induced homomorphism

$$
\psi_{*}: \pi_{1}(\langle\mathbf{x}\rangle) \longrightarrow \pi_{1}(\langle\mathbf{x} ; \mathbf{r}\rangle)
$$

Received June 5, 2002; in revised form June 23, 2003. 
is trivial. The main unsolvabilty result belong to Boone [4] and Novikov [12] which states that there exists a finitely presented group with unsolvable word problem.

As a natural extension of the above formulation of the word problem, for a finite connected 3-complex $\mathscr{K}$, one can ask whether for any arbitrary element of the second homotopy group $\pi_{2}(\mathscr{P})$, its image under the inclusion induced homomorphism

$$
\theta_{*}: \pi_{2}(\mathscr{P}) \longrightarrow \pi_{2}(\mathscr{K})
$$

is trivial.

Since a 2 -complex with a single 0 -cell can be regarded just as a presentation, we can consider a 3-complex with a single 0-cell, which is known as a 3presentation. A 3-presentation $\mathscr{K}$ is a triple

$$
\langle\mathbf{x} ; \mathbf{r} ; \mathbf{Y}\rangle
$$

where $\mathbf{Y}$ is a set of spherical pictures over $\mathscr{P}$. We say that $\mathscr{K}$ is finite if $\mathbf{x}, \mathbf{r}$ and $\mathbf{Y}$ are all finite. In fact this definition comes from the definition of an extension group presentation, defined by Fenn [9].

By using [3, Theorem 1.6] and [14, Theorem 2.6], we can formulate the generalised identity problem as "is there an algorithm to decide for any spherical picture $\mathbf{P}$ over $\mathscr{P}=\langle\mathbf{x} ; \mathbf{r}\rangle$ whether $\mathbf{P}$ is equivalent (relative to $\mathbf{Y}$ ) to the empty picture, for a given finite 3-presentation $\mathscr{K}=\langle\mathbf{x} ; \mathbf{r} ; \mathbf{Y}\rangle$ ?"

In this paper we work on the generalised identity problem which is the analogue of the one dimension higher of the word problem, by using the above contruction on 3-complexes.

Thus the main result of this paper is the following.

THEOREM 1.1. There is a finite 3-presentation $\mathscr{K}$ with unsolvable generalised identity problem. Furthermore, since $\mathscr{K}$ can be chosen, the word problem for the presentation $\mathscr{P}$ is solvable.

REMARK 1.2. It is well known that the word problem plays a central role in decision problems. Thus one can generate the above theorem to the global properties such as the $p$-Cockcroft properties or the efficiency. The reader can find the details and some applications of the $p$-Cockcroft property and the efficiency, for instance, in [6], [7].

By the paper written by Otto ([13]) it has been stated that the word problem for groups is reducible to the conjugacy problem, and so the decidability of the conjugacy problem implies the decidability of the word problem but the situation is different for monoids. As a result of this, he showed that the word problem and the conjugacy problem are independent of one another for monoids. Therefore, for a future project, by using the same technique and similar 
construction as in our this paper, the same result (Theorem 1.1 above) has been obtained seperately for monoids in the joint paper [8] written by Cevik and Ates.

\section{Preliminaries}

In this section we recover some basic concepts briefly which are needed in the next section. We refer to the reader [14] for the details.

\subsection{Spherical pictures}

Let $G$ be a finitely presented group with the presentaton $\mathscr{P}$, as in (1). If we regard $\mathscr{P}$ as a 2-complex with single 0 -cell whose 1-cells are in bijective correspondence with the elements of $\mathbf{x}$, and whose 2-cells are attached by the boundary path determined by the spelling of the correponding element of $\mathbf{r}$ in the standard way, then $G$ is just the fundamental group of $\mathscr{P}$. Thus there is also, of course, the second homotopy group $\pi_{2}(\mathscr{P})$ of $\mathscr{P}$, which is a left $Z G$-module. The elements of $\pi_{2}(\mathscr{P})$ can be represented by geometric configurations called spherical pictures. These are described in detail in [3] and [14]. In this paper we need only one basepoint on each disc of our pictures (so we will actually use $*$-pictures, as described in Section 2.4 of [14]). Also, as described in [14], there are certain operations on spherical pictures.

For a non-spherical picture $\mathrm{Q}$ over $\mathscr{P}$, we have the term boundary label which is the word read off by travelling around boundary of $\mathrm{Q}$ once in the clockwise direction starting from the basepoint of the Q. If a word $W$ in $\mathbf{x} \cup \mathbf{x}^{-1}$ represents an element in the normal closure $N$ of $\mathbf{r}$ in $F(\mathbf{x})$, then it is easy to construct a based picture over $\mathscr{P}$ with boundary label $W$. So there is the following pictorial version of the "van Kampen lemma" [5], [10].

Proposition 2.1 ([3, Theorem 1.4]). A word $W$ in $\mathbf{x} \cup \mathbf{x}^{-1}$ represents an element of $N$, that is $\bar{W}=1$ in $G(\mathscr{P})$, if and only if there is a based picture over $\mathscr{P}$ with boundary label $W$.

Suppose $\mathbf{Y}$ is a collection of spherical pictures over $\mathbf{P}$. Then, by [14], one can define the additional operation on spherical pictures. Allowing this additional operation leads to the notion of equivalence ( $\mathrm{rel} \mathbf{Y}$ ) of spherical pictures. We note that an example of using pictures can be found in [6].

In [14], Pride also proved the following important result about the equivalence of spherical pictures.

THEOREM 2.2. The elements $\langle\mathbf{P}\rangle(\mathrm{P} \in \mathbf{Y})$ generate $\pi_{2}(\mathscr{P})$ as a module if and only if every spherical picture is equivalent ( $\mathrm{rel} \mathbf{Y})$ to the empty picture.

Therefore it is easy to see that if the elements $\langle\mathbf{P}\rangle(\mathbf{P} \in \mathbf{Y})$ generate $\pi_{2}(\mathscr{P})$ then we say that $\mathbf{Y}$ generates $\pi_{2}(\mathscr{P})$. 


\subsection{Projective resolutions}

Let

$$
\longrightarrow P_{n} \longrightarrow \cdots \longrightarrow P_{2} \longrightarrow P_{1} \longrightarrow P_{0} \longrightarrow \mathrm{Z} \longrightarrow 0
$$

be any arbitrary projective resolution of the trivial $G$-module $Z$. This resolution is called $n$-finite if $P_{0}, P_{1}, \ldots, P_{n}$ are finitely generated. We say that $G$ is of type $F P_{n}(0 \leq n<\infty)$ if $G$ has an $n$-finite projective resolution. If the group $G$ is given by a presentation $\mathscr{P}$, as in (1), then we have the Lyndon resolution

$$
0 \longrightarrow \pi_{2}(\mathscr{P}) \stackrel{\mu}{\longrightarrow} P_{2} \longrightarrow P_{1} \longrightarrow \mathrm{Z} G \longrightarrow \mathrm{Z} \longrightarrow 0
$$

where $P_{1}$ and $P_{2}$ are the free left $Z G$-modules with basis $\left\{e_{x}: x \in \mathbf{x}\right\}$ and $\left\{e_{R}: R \in \mathbf{r}\right\}$, respectively. By [14], the embedding $\mu$ is given as follows. Let $\langle\mathrm{P}\rangle \in \pi_{2}(\mathscr{P})$ and suppose $\mathrm{P}$ has discs $\Delta_{1}, \Delta_{2}, \ldots, \Delta_{n}$, which each of them has single basepoint, with clockwise label $R_{1}^{\varepsilon_{1}}, R_{2}^{\varepsilon_{2}}, \ldots, R_{n}^{\varepsilon_{n}}$ respectively $\left(R_{i} \in \mathbf{r}\right.$, $\varepsilon_{=} \pm 1$ and $\left.i=1,2, \ldots, n\right)$. Let $\gamma_{i}$ be a transverse path from the basepoint of $\mathrm{P}$ to the basepoint of each $\Delta_{i}$. Let $W_{i}$ be the label on $\gamma_{i}$. Then

$$
\mu(\langle\mathrm{P}\rangle)=\sum_{i=1}^{n} \varepsilon_{i} g_{i} e_{R_{i}}
$$

where $g_{i}$ is the element of $G$ represented by $W_{i}$.

\section{Proof of the main theorem}

To construct this section we will pick a special group $M$ (see below) and then we will use it to obtain a finite 3-presentation $\mathscr{K}$. By taking an arbitrary word $W$ on the generating set of $M$, we will consider the set of $\mathbf{P}_{W}$ (where each $\mathrm{P}_{W}$ is a spherical picture over the presentation of $M$ ) and show that $\mathrm{P}_{W}$ is equivalent to the empty picture if and only if $W$ defines an element of a special normal subgroup of $M$, say $L$ (see below). Thus we can conclude that $\mathscr{K}$ has unsolvable generalised identity problem since, by the assumption on $L$, the subgroup word problem for $L$ in $M$ is unsolvable.

Let $M$ be finitely presented group defined by $\mathscr{P}_{M}=\langle\mathbf{x} ; \mathbf{r}\rangle$ such that

1) the word problem for the group $M$ is solvable,

2) $\pi_{2}\left(\mathscr{P}_{M}\right)$ is finitely generated,

3) there exists a finitely generated normal subgroup $L$ of $M$ such that the subgroup word problem for $L$ in $M$ is unsolvable.

We should note that the above analogue is a direct combination of the Novikov-Boone Theorem ([4], [12]) and the so-called Rips construction [15]. 
We could take the group of Miller [2, Corollary 1] for a specific example of a such group. One of the fact for this choise is the Miller group has an aspherical presentation and so $\pi_{2}\left(\mathscr{P}_{M}\right)=0$. Therefore the second condition holds.

Let $\mathrm{Z}_{2}$ be cyclic group of order 2 with a presentation $\mathrm{P}_{\mathrm{Z}_{2}}=\left\langle s ; s^{2}\right\rangle$ and let $T$ be a group $M \times \mathrm{Z}_{2}$ given by the presentation

$$
\mathscr{P}_{T}=\left\langle\mathbf{x}, s ; \mathbf{r}, s^{2},[x, s](x \in \mathbf{x})\right\rangle .
$$

We note that $T$ has solvable word problem since $M$ and $Z_{2}$ have both solvable word problem.

We need the following definition for the proof.

Definition 3.1. Let $x_{1}^{\varepsilon_{1}} \ldots x_{j}^{\varepsilon_{j}} x_{j+1}^{\varepsilon_{j+1}} \ldots x_{n}^{\varepsilon_{n}}$ be a word on $\mathbf{x}$. Then a commutator picture $\mathrm{D}_{x_{1}^{\varepsilon_{1}} \ldots x_{j}^{\varepsilon_{j}}} x_{j+1}^{\varepsilon_{j+1} \ldots} \ldots x_{n}^{\varepsilon_{n}}$ is a picture over $\langle\mathbf{x}, s ;[x, s](x \in \mathbf{x})\rangle$ of the form as depicted in Figure 1(a). Moreover, if $\mathrm{D}_{x_{1}^{\varepsilon_{1}} \ldots x_{j}^{\varepsilon_{j}} x_{j+1}^{\varepsilon_{j+1}} \ldots x_{n}^{\varepsilon_{n}}}$ is a picture over $\left\langle\mathbf{x}, s ; s^{2},[x, s](x \in \mathbf{x})\right\rangle$, then it is equivalent to a picture as shown in Figure 1(b).

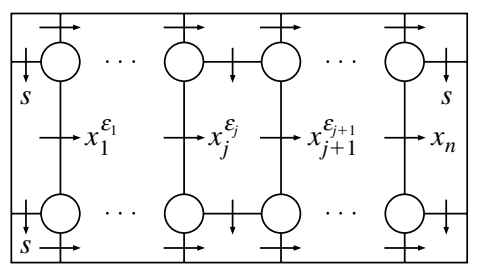

(a)

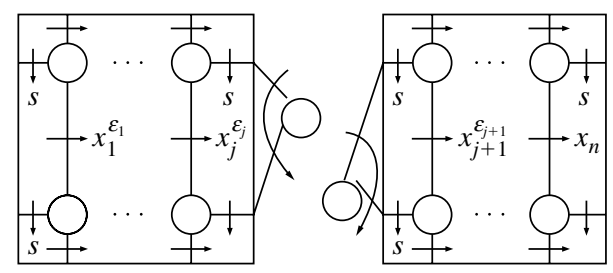

(b)

FIGURE 1

Suppose that $\mathbf{w}=\left\{w_{1}, w_{2}, \ldots, w_{n}\right\}$ is a set of words on $\mathbf{x}$ which represents a finite set of generators of $L$. Let $\mathbf{Y}_{\mathbf{1}}$ be a finite set of spherical pictures which generates $\pi_{2}\left(\mathscr{P}_{M}\right)$, and for each $R \in \mathbf{r}$, let $\mathbf{Y}_{2}$ be the finite set of spherical pictures (see Figure 2(a)) over the presentation $\mathscr{P}_{T}$, as given in (3).

Also let $\mathbf{Y}_{3}$ consists of the single picture as drawn in Figure 2(b). Finally, for each $w_{i} \in \mathbf{w}$, let $\mathbf{Y}_{4}$ be a finite set of spherical pictures over $\mathscr{P}_{T}$ of the form as shown in Figure 3(a).

We note that the subpicture $\mathrm{D}_{i}$ is a commutator picture and fixed over the presentation $\langle\mathbf{x}, s ;[x, s](x \in \mathbf{x})\rangle$.

Let $\mathbf{Y}=\mathbf{Y}_{1} \cup \mathbf{Y}_{2} \cup \mathbf{Y}_{3} \cup \mathbf{Y}_{4}$. Since each $\mathbf{Y}_{j}(1 \leq j \leq 4)$ is finite, $\mathbf{Y}$ is finite. Therefore we have a finite 3-presentation

$$
\mathscr{K}=\left\langle\mathbf{x}, s ; \mathbf{r}, s^{2},[x, s](x \in \mathbf{x}) ; \mathbf{Y}\right\rangle
$$




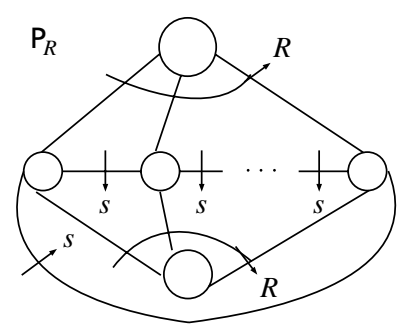

(a)

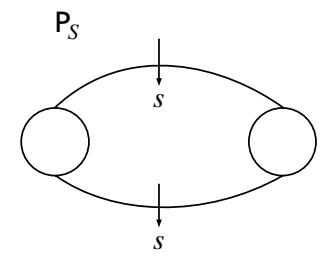

(b)

FIGURE 2

$$
\mathbf{P}_{w_{i}}
$$

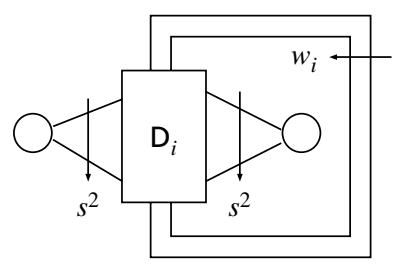

(a)

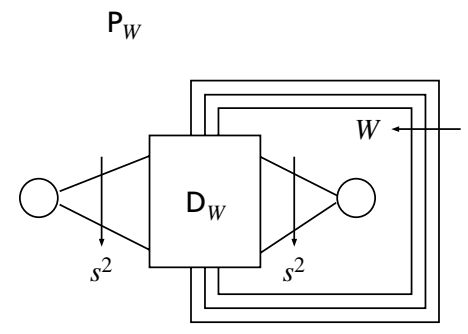

(b)

FIGURE 3

such that the underlying presentation $\mathscr{P}_{T}$ has solvable word problem.

Now suppose that $M$ is finitely presented group defined by $\mathscr{P}_{M}$ as above. For any word $W$ on $\mathbf{x}$, let $\mathbf{P}_{W}$ be a spherical picture of the form as shown in Figure $3(\mathrm{~b})$. As in $\mathrm{D}_{i}$, we again note that the commutator subpicture $\mathrm{D}_{W}$ is fixed over the presentation $\langle\mathbf{x}, s ;[x, s](x \in \mathbf{x})\rangle$.

Now, we will show that $\mathbf{P}_{W}$ is equivalent to the empty picture (relative to Y) if and only if $W$ defines an element of $L$, and hence $\mathscr{K}$ has unsolvable generalised identity problem since the subgroup word problem for $L$ in $M$ is unsolvable. Let us start to show this with the sufficiency part. So let us assume that $W$ defines an element of $L$. Then $\bar{W}=\overline{w_{1}^{\varepsilon_{1}} w_{2}^{\varepsilon_{2}} \ldots w_{n}^{\varepsilon_{n}}}$ in $M$ for some $w_{i}$ 's which belong to $\mathbf{w}$ and $\varepsilon_{i}= \pm 1$. Thus, by Proposition 2.1, there is a picture $\mathrm{Q}$ over $\mathscr{P}_{M}=\langle\mathbf{x} ; \mathbf{r}\rangle$ with boundary label $w_{1}^{\varepsilon_{1}} w_{2}^{\varepsilon_{2}} \ldots w_{n}^{\varepsilon_{n}} W^{-1}$. Now let us consider the picture $\mathrm{P}_{\mathrm{Q}}^{1}$ as shown in Figure 4(a). We note that, by cancelling pair operation (see [14]), $\mathrm{P}_{\mathrm{Q}}^{1}$ is equivalent to the picture $\mathrm{P}_{\mathrm{Q}}^{2}$, as depicted in Figure 4(b). By [1], the set $\mathbf{Y}_{1} \cup \mathbf{Y}_{2}$ generates $\pi_{2}(\langle\mathbf{x}, s ; \mathbf{r},[x, s](x \in \mathbf{x})\rangle)$. Then, by Theorem 14, $\mathrm{P}_{\mathrm{Q}}^{2}$ is equivalent (relative to $\mathbf{Y}_{1} \cup \mathbf{Y}_{2}$ ) to the empty picture and so is $\mathrm{P}_{\mathrm{Q}}^{1}$. 


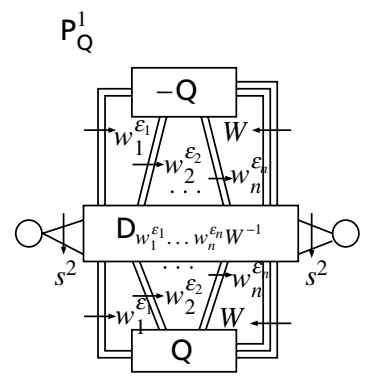

(a)

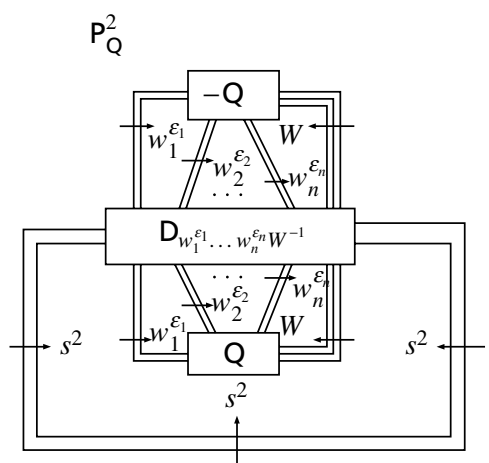

(b)

FIGURE 4

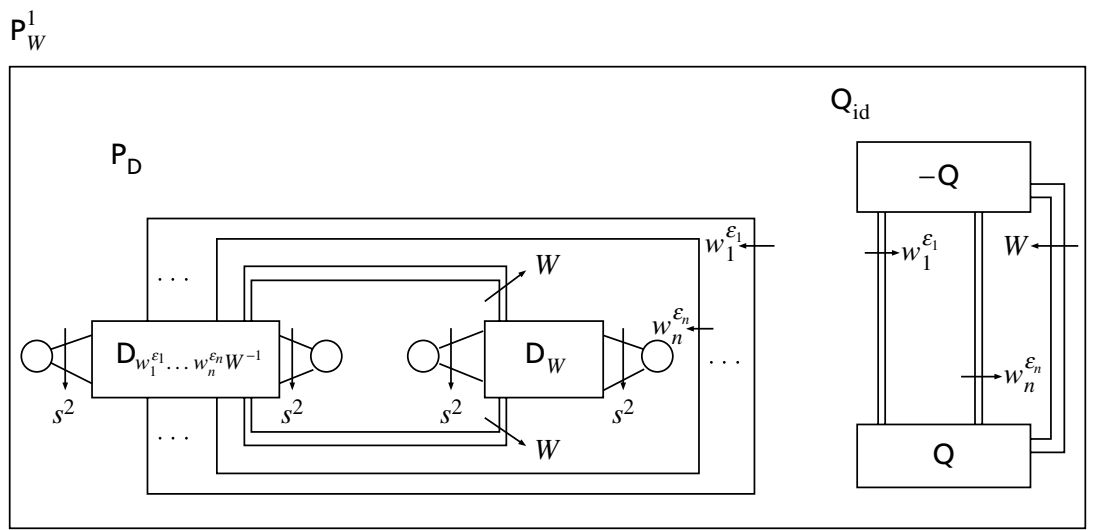

FIGURE 5

Now, by inserting $\mathrm{P}_{\mathrm{Q}}^{1}$ to the left side of $\mathrm{P}_{W}$ and performing some bridge move operations (see [14]), we have the picture $P_{W}^{1}$, as in Figure 5, which contains two subpictures $P_{D}$ and $Q_{i d}$.

Since the subpicture $Q_{i d}$ is equivalent to the empty picture (we can think it as a cancelling pair), we delete it. Moreover, by Definition 3.1, the subpicture $P_{D}$ becomes the picture $P_{D}^{\prime}$ as in Figure 6. In this picture we can delete the subpicture $S_{W}$, depicted in Figure 7, since it is equivalent to the empty picture (by applying some bridge move and cancelling pair operations on it).

Furthermore, by applying a sequence of Definition 3.1, we obtain the picture $\mathbf{P}_{W}^{2}$ (see Figure 8) which is equivalent (relative to $\mathbf{Y}_{3}$ ) to the empty picture.

These all above processes give us that the picture $\mathbf{P}_{W}$ is equivalent (relative 


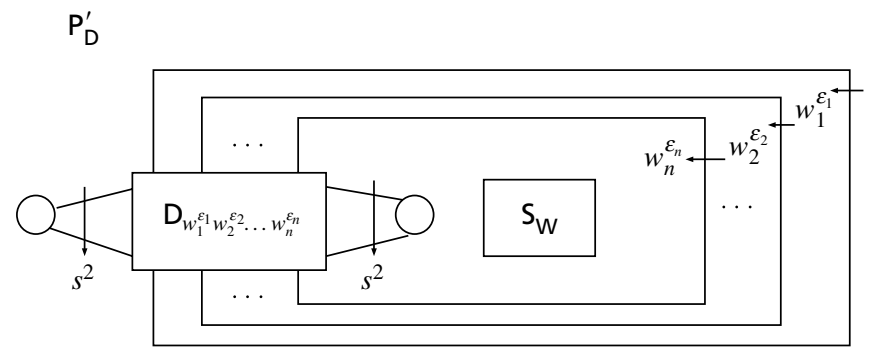

FIGURE 6

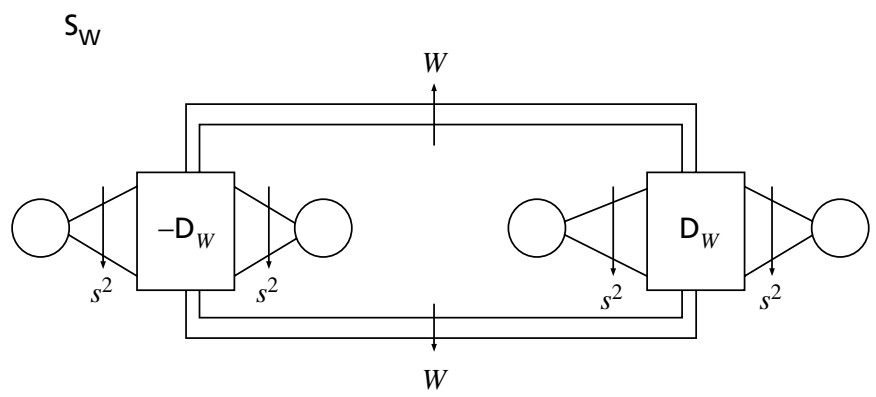

FIGURE 7

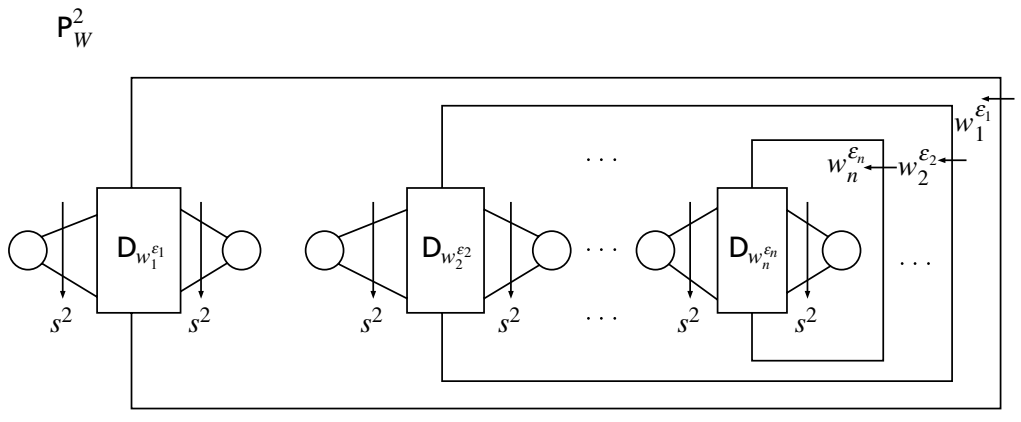

Figure 8

to $\mathbf{Y}$ ) to the empty picture, as required.

For the necessity part of the above process, we will show that if $W$ does not define any element of $L$, then $\mathbf{P}_{W}$ is not equivalent to the empty picture (relative to $\mathbf{Y}$ ) over $\mathscr{P}_{T}$.

Suppose that $\mathbf{P}_{W}$ can be obtained from $\mathbf{Y}$. Let $P_{2}$ be the free $\mathbf{Z T}$-module with basis $\left\{e_{R}: R \in \mathbf{r}\right\} \cup\left\{e_{s^{2}}\right\} \cup\left\{e_{[x, s]}: x \in \mathbf{x}\right\}$. By considering the exact 
sequence, as given in (2), we will determine the image of $\mathrm{P}_{W}$ in $P_{2}$. We recall that each $\gamma$ (as used in below) defines a transverse path from the basepoint of the concerned picture to the basepoint of each discs of this picture.

Let us take $P_{2}=\mathrm{ZT} e_{s^{2}} \oplus P_{2}^{\prime}$, where $P_{2}^{\prime}$ is the free $\mathrm{ZT}$-module with the above basis excluding $\left\{e_{S^{2}}\right\}$. Then the image of $\mathrm{P}_{W}$ in $P_{2}$ is

$$
\gamma_{W}=(\bar{W}-1) e_{s^{2}}+\lambda_{W},
$$

for some $\lambda_{W} \in P_{2}^{\prime}$, and the image of $\mathbf{P}_{w_{i}}\left(\mathbf{P}_{w_{i}} \in \mathbf{Y}_{4}\right)$ is

$$
\gamma_{i}=\left(\overline{w_{i}}-1\right) e_{s^{2}}+\lambda_{i},
$$

for some $\lambda_{i} \in P_{2}^{\prime}$. Also let the image of each $\mathrm{P}_{R}(R \in \mathbf{r})$ be $\gamma_{R}$ and the image of $\mathbf{B}\left(\mathbf{B} \in \mathbf{Y}_{1}\right)$ be $\gamma_{\mathbf{B}}$. We should note that $\gamma_{R}$ and $\gamma_{\mathrm{B}}$ contained in $P_{2}^{\prime}$.

By the assumption, since $\mathbf{P}_{W}$ is obtainable from $\mathbf{Y}$, we then have

$$
\gamma_{W}=\beta_{1} \gamma_{1}+\beta_{2} \gamma_{2}+\cdots+\beta_{n} \gamma_{n}+\alpha_{0} \gamma_{s^{2}}+\sum_{R \in \mathbf{r}} \alpha_{R} \gamma_{R}+\sum_{\mathbf{B} \in \mathbf{Y}_{1}} \alpha_{\mathrm{B}} \gamma_{\mathrm{B}},
$$

where each of the $\alpha$ and $\beta$ belongs to $Z T$. Let us equate the coefficients of $e_{s^{2}}$. Then we get

$$
\bar{W}-1=\beta_{1}\left(\overline{w_{1}}-1\right)+\beta_{2}\left(\overline{w_{2}}-1\right)+\cdots+\beta_{n}\left(\overline{w_{n}}-1\right)+\alpha_{0}(\bar{s}-1) .
$$

After all, if we consider the induced ring homomorphism

$$
\mathrm{Z} T \longrightarrow \mathrm{Z} M \longrightarrow \mathrm{Z}(M / L), \quad \bar{x} \longmapsto \bar{x} \longmapsto \bar{x} L(x \in \mathbf{x}), \bar{s} \longmapsto 1 \longmapsto 1 L
$$

arising from the group homomorphism, then we have $\bar{W} L-1 L=0$. In other words $W$ defines the element $\bar{W}$ of $L$ which makes contradiction to our assumption.

Hence the result.

Final Remark 3.2. The finite 3-presentation that we produce in our main result (Theorem 1.1) can be taken to have as two-skeleton a presentation $\mathscr{P}$ for a group $G$ that not only has solvable word problem, but which is also of homological finiteness type $F_{\infty}$ : the group $G$ admits a $K(G, 1)$ with finitely many cells in each dimension. To identify a finite three-skeleton, we need only replace the set $\mathbf{Y}_{4}$ of spherical pictures $\mathbf{P}_{w_{i}}\left(w_{i} \in \mathbf{w}\right)$ with the analogous pictures $\mathbf{P}_{x}(x \in \mathbf{x})$.

ACKNOWLEDGEMENT. The author is grateful to the referee for many helpful suggestions, comments and for kind help in modifying the original version of the paper. 


\section{REFERENCES}

1. Baik, Y. G., and Pride, S. J., Generators of the second homotopy modules of presentations arising from groups constructions, preprint, University of Glasgow, 1992.

2. Baumslag, G., Miller III, C. F., and Short, H., Unsolvable problems about small cancellation and word hyperbolic groups, Bull. London Math. Soc. 26 (1994), 97-101.

3. Bogley, W. A., and Pride, S. J., Calculating generators of $\pi_{2}$, in Two-dimensional homotopy and combinatorial group theory (C. Hog-Angeloni, W. Metzler and A. J. Sieradski, eds.), London Math. Soc. Lecture Note Ser. 197 (1993), CUP, 157-188.

4. Boone, W. W., Certain simple unsolvable problems in group theory I, II, III, IV, V, VI, Nederl. Akad. Wetensch Proc. Ser. A 57 (1954), 231-237, 492-497; 58 (1955), 252-256, 571-577; 60 (1957), 22-27, 227-232.

5. Brown, R., and Huebschmann, J., Identities among relations, in: Low-Diemensional Topology (R. Brown and T. L. Thickstun, eds.), London Math. Soc. Lecture Note Ser. 48 (1982), CUP, 153-202.

6. Çevik, A. S., The p-Cockcroft property of central extensions of groups, Comm. Algebra 29(3) (2001), 1085-1094.

7. Çevik, A. S., The efficiency of standard wreath product, Proc. Edinburgh Math. Soc. 43 (2000), 415-423.

8. Çevik, A. S., and Ateş, F., One dimension higher of the word problem for monoids, preprint, Balikesir University, 2003.

9. Fenn, R. A., Techniques in Geometric Group Theory, London Math. Soc. Lecture Note Ser. 57 (1983), CUP.

10. Lyndon, R. C., and Schupp, P. E., Combinatorial Group Theory, Springer-Verlag (1977).

11. Miller III, C. F., Decision problems for groups - survey and reflections, in Algorithms and classification in combinatorial group theory (G. Bumslag and C. F. Miller III eds.), Math. Sci. Res. Inst. Publ. 23 (1992), 1-59.

12. Novikov, P. S., On the algorithmic unsolvability of the word problem in group theory, Trudy Mat. Inst. Steklov 44 (1955), 1-143.

13. Otto, F., Conjugacy in monoids with a special Church-Rosser presentation is decidable, Semigroup Forum 29 (1984), 223-240.

14. Pride, S. J., Identities among relations of group presentations, in Group theory from geometrical viewpoint-Trieste 1990, World Scientific Publishing, Singapore (1991), 687-717.

15. Rips, E., Subgroups of small cancellation groups, Bull. London Math. Soc. 14(1) (1982), $45-47$.

BALIKESIR UNIVERSITESI

FEN-EDEBIYAT FAKULTESI

MATEMATIK BOLUMU

10100, BALIKESIR

TURKEY

E-mail: scevik@balikesir.edu.tr 